\title{
An $L^{\infty}$ Bound for a Time-discrete Regularization of a Forward-backward Parabolic Equation
}

\author{
NorA E. MUler
}

ABSTRACT. In this paper we consider a time discrete regularization of a backward-forward parabolic equation that is not necessarily well posed. This time-discrete scheme in $\mathbb{R}^{n}$ can be formulated as

$$
v_{m+1}=K_{\Delta t} * v_{m}+\Delta t K_{\Delta t} * \sum_{i, j=1}^{n} f_{i j}\left(v_{m}, x\right),
$$

with $f_{i j}(v, \cdot)$ constant when $v$ is large, $K$ a positive kernel with compact support,

$$
K_{s}(x)=\frac{1}{s^{n / 2}} K\left(\frac{x}{s^{1 / 2}}\right),
$$

$s>0$. The result we obtain is a uniform bound for $\left(v_{m}\right)_{m \in \mathbb{N}}$ independent of $\Delta t>0$.

1. Introduction. This paper is mainly concerned with the existence of an $L^{\infty}$ bound for the following time-discrete scheme in $\mathbb{R}^{n}$,

$$
\left\{\begin{array}{l}
v_{m+1}=K_{\Delta t} * v_{m}+\Delta t K_{\Delta t} * \sum_{i, j=1}^{n} \frac{\partial^{2}}{\partial x_{i} \partial x_{j}} f_{i j}\left(v_{m}, x\right) \\
v_{0} .
\end{array}\right.
$$

For the kernel $K_{s}, s>0$ we assume $K_{s}(x)=\left(1 / s^{n / 2}\right) K\left(x / s^{1 / 2}\right)$, where

$$
K \in C_{c}^{3}\left(\mathbb{R}^{n}\right), \quad K \geq 0, \quad \int_{\mathbb{R}^{n}} K(x) d x=1
$$


and

$$
\int_{\mathbb{R}^{n}} x_{i} K(x) d x=0, \quad \int_{\mathbb{R}^{n}} x_{i} x_{j} K(x) d x=\delta_{i j}, \quad i, j=1 \ldots n .
$$

Note that $K$ can be regarded as a density function of a random vector with all the first moments being zero and the identity as the covariance matrix.

The functions $f_{i j} i, j=1 \ldots n$ are constant outside a bounded interval. To fix the notation we consider this interval as $[-1,1]$, and we assume that

$$
f_{i j}: \mathbb{R}^{n+1} \rightarrow \mathbb{R} i, j=1 \ldots n \text { is Lipschitz continuous, }
$$

$f_{i j}(\lambda, x)=C_{1}^{i j}$ where $\lambda \geq 1$, and $f_{i j}(\lambda, x)=C_{2}^{i j}$ where $\lambda \leq-1 i, j=1 \ldots n$, uniformly in $x \in \mathbb{R}^{n}$.

For the initial condition we assume that

$$
v_{0} \in L^{\infty}, \nabla v_{0} \in\left(L^{\infty}\right)^{n} .
$$

Notice that for a given fixed time, the repeated convolution of the positive kernel $K_{\Delta t}$ with itself tends to the heat kernel as $\Delta t \rightarrow 0$. Therefore, at the limit $(\Delta t \rightarrow 0)$ we formally get (1.6) below:

$$
\begin{cases}v_{t}=\Delta v+\sum_{i, j=1}^{n} \frac{\partial^{2}}{\partial x_{i} \partial x_{j}} f_{i j}(v, x) & \text { in } x \in \mathbb{R}^{n}, t>0 . \\ v(x, 0)=v_{0}(x) & \end{cases}
$$

For this reason, (1.1) can be seen as a time-discrete regularization of (1.6), that is, an initial-value problem that could be ill-posed depending on the monotonicity of the second-order operator. There is only one result in the one-dimensional case that ensures the existence of infinitely many solutions with $f=f_{11}$ piecewise linear (see Hollig-Nohel [HN]).

The main result of this paper is as follows:

Theorem 1. Let $\left(v_{m}\right)_{m \in \mathbb{N}}$ be solutions to (1.1), satisfying (1.2)-(1.4). Then, there is a positive constant $c$ such that

$$
\left\|v_{m}\right\|_{L^{\infty}} \leq c, \quad \Delta t>0, \quad m \in \mathbb{N} .
$$

Although this result does not ensure convergence, it gives a certain notion of stability as $\Delta t \rightarrow 0$. Theorem 1 is analogous to getting a uniform $L^{\infty}$ bound for the following rescaled problem in $\mathbb{R}^{n}$ :

$$
\left\{\begin{array}{l}
u_{m+1}=K * u_{m}+K * \sum_{i, j=1}^{n} \frac{\partial^{2}}{\partial x_{i} \partial x_{j}} f_{i j}\left(u_{m}, x\right) \\
u_{0}
\end{array}\right.
$$


where $u_{m}(x)=v_{m}(\sqrt{\Delta t} x)$. We can rewrite (1.8) as

$$
\left\{\begin{array}{l}
u_{m+1}=K^{(m+1)} * u_{0}+\sum_{\ell=1}^{m+1} K^{(\ell)} * \sum_{i, j=1}^{n} \frac{\partial^{2}}{\partial x_{i} \partial x_{j}} f_{i j}\left(u_{m-\ell+1}, x\right), \\
u_{0},
\end{array}\right.
$$

where $K^{(m)}$ is the $m$-th convolution of $K$ with itself.

The organization of the paper is as follows.

In Section 2, we prove that the gradient of the solutions of (1.8) has an $L^{\infty}$ bound, uniformly in $m \in \mathbb{N}$. For the proof we use the fact that $K^{(m)}$ approaches the heat kernel as $m \rightarrow+\infty$ (see for instance Durrett [D], Feller [Fe], and many others). Roughly speaking, this means that $K^{(m)}$ behaves as the heat kernel for $m$ large. In order to get the result, one must assume that $f_{i j}$ is bounded.

In Section 3, we prove first that $\left(u_{m+1}-u_{m}\right)$, namely the difference between two consecutive solutions of (1.8), has a bound in $L^{\infty}$ uniformly in $m \in \mathbb{N}$. We then obtain that the solutions of (1.8) have a bound in $L^{\infty}$ uniformly in $m \in \mathbb{N}$ and, as a consequence, we get the result of Theorem 1. In this section we use for the first time the compact support of $K$ (in the previous section an exponential decay at infinity would be enough).

Note: The Cahn-Hilliard equation (see for instance Cahn $[\mathrm{C}]$, Pego $[\mathrm{P}]$, Slemrod $[\mathrm{S}]$ ) can be seen as a fourth-order regularization of (1.6). In a previous paper (see Caffarelli-Muler $[\mathrm{CM}]$ ) we obtained an $L^{\infty}$ bound for the solutions of Cahn-Hilliard. Notice that in $[\mathrm{CM}]$ and in this paper we succeed in proving the existence of a uniform bound for regularizations of (1.6), even though we cannot demonstrate the existence of solutions in the limit.

\section{Notation.}

(1) $L^{p}=L^{p}\left(\mathbb{R}^{n}\right), 1 \leq p \leq+\infty$.

(2) $\delta_{i j}= \begin{cases}1 & i=j \\ 0 & i \neq j\end{cases}$

(3) The one star $(*)$ denotes the space convolution in $\mathbb{R}^{n}$.

(4) $B_{a}\left(x_{0}\right)=\left\{x /\left|x-x_{0}\right|<a\right\}$

(5) In Section 2 , given a function $g: \mathbb{R}^{n} \rightarrow \mathbb{R}$, we denote

$$
D^{\alpha} g(x)=\frac{\partial^{|\alpha|}}{\partial x_{1}^{\alpha_{1}} \ldots \partial x_{n}^{\alpha_{n}}} g(x),
$$

where $\alpha \in \mathbb{N}_{0}^{n}$ and $|\alpha|=\sum_{i=1}^{n} \alpha_{i}$ 
2. Bounds for the gradient. Let $u_{m}$ be the solution to (1.8). In this section we shall prove that $\nabla u_{m}$ have an $L^{\infty}$ bound uniformly in $m \in \mathbb{N}$.

The characteristic function $\varphi$ of the distribution function with density $K$ can be written as

$$
\varphi(x)=\int_{\mathbb{R}^{n}} e^{i x \cdot \xi} K(\xi) d \xi
$$

Hence,

$$
K^{(m)}(x)=\frac{1}{(2 \pi)^{n}} \int_{\mathbb{R}^{n}} e^{-i x \cdot \xi} \varphi^{m}(\xi) d \xi
$$

First, let us state some elementary results.

Lemma 1. For $x \in \mathbb{R}^{n}$ the following holds:

(i) $|\varphi(x)|<1 \quad x \neq 0$ and $\varphi(0)=1$;

(ii) $\lim _{\ell \rightarrow+\infty}\left|\varphi^{\ell-k}(x / \sqrt{\ell})\right|=e^{-|x|^{2} / 2}, k \in \mathbb{N}$;

(iii) Given $\alpha \in \mathbb{N}_{0}^{n}$, there is a positive constant c such that

$$
\left|D_{x}^{\alpha} \varphi(x)\right| \leq c
$$

(iv) There is a positive constant $c$ such that

$$
|\nabla \varphi(x)| \leq c|x|
$$

(v) There is a positive constant $c$ such that

$$
|\varphi(x)| \leq c \prod_{i=1}^{n} \frac{1}{\left|x_{i}\right|^{3}}
$$

Proof.

(i) From the definition of $\varphi$ it follows that $|\varphi(x)| \leq 1$ in $\mathbb{R}^{n}$ and $\varphi(0)=1$.

We should now see that the strict inequality holds for $x \neq 0$. Let us assume that there exists $\bar{x} \in \mathbb{R}^{n}$ such that $\varphi(\bar{x})=1$. Hence,

$$
\int_{\mathbb{R}^{n}} e^{i \bar{x} \cdot \xi}(K(\xi)-1) d \xi=\varphi(\bar{x})-1=0 .
$$

In particular,

$$
\bar{x} \cdot \xi=k_{\xi} \pi, \quad \forall \xi \in B_{\delta}(a) \subset \operatorname{supp}(K), \quad k_{\xi} \in \mathbb{Z}
$$

and this implies $\bar{x}=0$. 
(ii) From the assumptions on $K$ and the definition of $\varphi$, we have

$$
\varphi(0)=1, \quad \nabla \varphi(0)=0 \quad \text { and } \quad \frac{\partial^{2} \varphi(0)}{\partial x_{i} \partial x_{j}}=-\delta_{i j}
$$

Therefore,

$$
\varphi\left(\frac{x}{\sqrt{\ell}}\right)^{\ell-k}=\left(1-\frac{|x|^{2}}{2 \ell}+o\left(\frac{|x|^{2}}{\ell}\right)\right)^{\ell} \varphi\left(\frac{x}{\sqrt{\ell}}\right)^{-k} \underset{\ell \rightarrow+\infty}{\stackrel{-|x|^{2} / 2}{\longrightarrow}} e^{-}
$$

(iii) and (iv) follow from the compact support of $K$ and $\nabla \varphi(0)=0$. In fact, it would be enough to assume $K$ with an exponential decay at infinity.

(v) Using $K \in C_{c}^{3}\left(\mathbb{R}^{n}\right)$, we integrate (2.1) three times by parts and we get the result.

Now, we need some technical lemmas.

Lemma 2. There is a constant $c>0$ such that

$$
\left|D_{x}^{\alpha} \varphi^{\ell}\left(\frac{x}{\sqrt{\ell}}\right)\right| \leq c \sum_{k=1}^{s}\left|\varphi^{\ell-k}\left(\frac{x}{\sqrt{\ell}}\right)\right|\left(1+|x|^{k}\right), \quad \forall \ell \geq|\alpha|,
$$

where $s=\min (|\alpha|, \ell), \alpha_{i} \in\{0,1,2\}, i=1 \ldots n$.

Proof. Opening $D_{x}^{\alpha} \varphi^{\ell}(x / \sqrt{\ell})=D_{x}^{\alpha} \prod_{j=1}^{\ell} \varphi(x / \sqrt{\ell})$, for each $1 \leq k \leq s$ we have a summation such that the typical term becomes

$$
\ell^{-|\alpha| / 2}\left(\begin{array}{l}
\ell \\
k
\end{array}\right)\left(\begin{array}{c}
k \\
k_{1}
\end{array}\right)\left(k-k_{1}\right) ! k ! \varphi(y)^{\ell-k} \prod_{j=1}^{k} D_{y}^{\alpha(j)} \varphi(y),
$$

where $y=x / \sqrt{\ell}, 0 \leq k_{1} \leq k, \sum_{j=1}^{k} \alpha(j)_{i}=\alpha_{i}, i=1 \ldots n,|\alpha(j)| \geq 2, j=1 \ldots k_{1}$, $|\alpha(j)|=1, j=k_{1}+1 \cdots k$.

Using Lemma 1 (iii) and (iv) and taking into account that $|\alpha| \geq k_{1}+k$, we can obtain that (2.7) is bounded by

$$
\begin{aligned}
& \ell^{-\left(|\alpha| / 2+k-k_{1}\right)}\left|\varphi\left(\frac{x}{\sqrt{\ell}}\right)^{\ell-k}\right|\left(\begin{array}{l}
\ell \\
k
\end{array}\right)\left(\begin{array}{c}
k \\
k_{1}
\end{array}\right)\left(k-k_{1}\right) ! k !|x|^{k-k_{1}} \\
\leq & d\left|\varphi\left(\frac{x}{\sqrt{\ell}}\right)^{\ell-k}\right|\left(|x|^{k}+1\right),
\end{aligned}
$$

where $d$ is a positive constant.

Hence, we get the result. 
Lemma 3. There is a constant $c>0$ that does not depend on $\ell \in \mathbb{N}$ such that

$$
\int_{\mathbb{R}^{n}}\left|P(x) \varphi\left(\frac{x}{\sqrt{\ell}}\right)^{\ell-k}\right| d x \leq c,
$$

where $0 \leq k \leq 2 n, k<\ell$ and $P: \mathbb{R}^{n} \rightarrow \mathbb{R}$ is a polynomial of degree at most one in each variable $x_{i}$.

Proof. We can split the integral as

$$
\int_{\mathbb{R}^{n}}\left|P(x) \varphi\left(\frac{x}{\sqrt{\ell}}\right)^{\ell-k}\right| d x=\sum_{i=1}^{3} \int_{I_{i}}\left|P(x) \varphi\left(\frac{x}{\sqrt{\ell}}\right)^{\ell-k}\right| d x
$$

where

$$
\begin{aligned}
& I_{1}=\left\{x /\left|x_{i}\right| \leq \sqrt{\ell} \varepsilon, i=1 \ldots n\right\}, \\
& I_{2}=\left\{x / \sqrt{\ell} \varepsilon \leq\left|x_{i}\right| \leq \ell^{2}, i=1 \ldots n\right\} \\
& I_{3}=\left\{x /\left|x_{i}\right| \geq \ell^{2}, i=1 \ldots n\right\} .
\end{aligned}
$$

The proof consists in estimating each term in the previous expression separately. From Lemma 1 , for all $x \in I_{1}$ and $\varepsilon$ small enough,

$$
\left|\varphi\left(\frac{x}{\sqrt{\ell}}\right)^{\ell-k}\right| \leq e^{-|x|^{2} / 4}
$$

In view of Lemma 1 (i), for all $x \in I_{2}$ there is a constant $0<q<1$ such that

$$
\left|\varphi\left(\frac{x}{\sqrt{\ell}}\right)\right| \leq q \text {. }
$$

Therefore,

$$
\int_{I_{2}}\left|P(x) \varphi\left(\frac{x}{\sqrt{\ell}}\right)\right|^{\ell-k} d x \leq q^{\ell-k} \int_{I_{2}}|P(x)| d x .
$$

and this last integral can be bounded by a polynomial with variable $\ell$.

Finally, from Lemma $1(\mathrm{v})$ and the definition of $I_{3}$, we have that there is a positive constant $c$ such that

$$
\begin{aligned}
\int_{I_{3}}\left|P(x) \varphi\left(\frac{x}{\sqrt{\ell}}\right)^{\ell-k}\right| d x & \leq c \int_{I_{3}}|P(x)| \prod_{i=1}^{n}\left(\frac{\sqrt{\ell}}{\left|x_{i}\right|}\right)^{3(\ell-k)} d x \\
& \leq c \int_{I_{3}}|P(x)| \prod_{i=1}^{n} \frac{1}{\left|x_{i}\right|^{(9 / 4)(\ell-k)}} d x
\end{aligned}
$$

that is bounded for $\ell$ large enough. Collecting the bounds $(2.10)-(2.13)$, we obtain the result of the lemma. 
Lemma 4. There is a constant $c>0$ that does not depend on $\ell$ such that

$$
\left\|\int_{\mathbb{R}^{n}}\left|P(\eta) \varphi^{\ell}\left(\frac{\eta}{\sqrt{\ell}}\right) e^{i \eta \cdot x} d \eta\right|\right\|_{L^{1}} \leq c
$$

where $P: \mathbb{R}^{n} \rightarrow \mathbb{R}$ is a polynomial of degree at most one in each variable $\eta_{i}$.

Proof. For $\ell \leq 2 n$, using the decay of $\varphi$, we can easily see that the result holds. Now, let us consider the case $\ell>2 n$.

Choose a function $\vartheta \in C_{c}^{1}$ such that

$$
\vartheta(\lambda) \leq 1 \quad \text { and } \quad \vartheta(\lambda)= \begin{cases}1 & \text { if }|\lambda| \leq 1 \\ 0 & \text { if }|\lambda| \geq 2\end{cases}
$$

we have

$$
1=\prod_{j=1}^{n}\left(\vartheta\left(x_{j}\right)+\bar{\vartheta}\left(x_{j}\right)\right), \quad \text { where } \bar{\vartheta}=1-\vartheta .
$$

Therefore, we can write the integral in (2.14) as

$$
\int_{\mathbb{R}^{n}} \prod_{j=1}^{n}\left(\vartheta\left(x_{j}\right)+\bar{\vartheta}\left(x_{j}\right)\right)\left|\int_{\mathbb{R}^{n}}\right| P(\eta) \varphi^{\ell}\left(\frac{\eta}{\sqrt{\ell}}\right)\left|e^{i \eta \cdot x} d \eta\right| d x .
$$

We open all the sums in products (after reordering $\left(\eta_{1}, \ldots, \eta_{n}\right),\left(x_{1}, \ldots, x_{n}\right)$ ) and the typical term becomes

$$
\int_{\mathbb{R}^{n}} \prod_{j=1}^{k} \bar{\vartheta}\left(x_{j}\right) \prod_{j=k+1}^{n} \vartheta\left(x_{j}\right)\left|\int_{\mathbb{R}^{n}}\right| P(\eta) \varphi^{\ell}\left(\frac{\eta}{\sqrt{\ell}}\right)\left|e^{i \eta \cdot x} d \eta\right| d x
$$

where $0 \leq k \leq n$. We integrate by parts two times with respect to the variables $\eta_{j}, 1 \leq j \leq k$ (if there are any, that is, when $k \neq 0$ ) and the integral can be bounded by a sum of terms that can be written as

$$
\int_{\mathbb{R}^{n}} \prod_{j=1}^{k} \frac{1}{x_{j}^{2}} \bar{\vartheta}\left(x_{j}\right) \prod_{j=k+1}^{n} \vartheta\left(x_{j}\right) \int_{\mathbb{R}^{n}}\left|\bar{P}(\eta) D_{\eta}^{\alpha} \varphi^{\ell}\left(\frac{\eta}{\sqrt{\ell}}\right)\right| d \eta d x,
$$

where $\bar{P}: \mathbb{R}^{n} \rightarrow \mathbb{R}$ are polynomials of degree at most one in each variable $\eta_{i}$, $\alpha_{i} \in\{0,1,2\} i=1 \ldots n,|\alpha| \leq 2 k$.

Hence, from Lemma 2 and Lemma 3 we get the desired result.

Now, let us state the main result of this section. 
Proposition 1. There is a constant $c>0$ that does not depend on $m \in \mathbb{N}$ such that

$$
\left\|\nabla u_{m}\right\|_{\left(L^{\infty}\right)^{n}} \leq c
$$

where $u_{m} m \in \mathbb{N}$ are solutions of (1.8).

Proof. From (1.9) we can write

$$
\begin{array}{r}
\frac{\partial}{\partial x_{k}} u_{m}=K^{(m)} * \frac{\partial}{\partial x_{k}} u_{0}+\sum_{\ell=1}^{m} K^{(\ell)} * \sum_{i, j=1}^{n} \frac{\partial^{3}}{\partial x_{i} \partial x_{j} \partial x_{k}} f_{i j}\left(u_{m-\ell}, \cdot\right), \\
k=1 \ldots n .
\end{array}
$$

Using Hölder's inequality and standard properties of the convolutions, one has

$$
\left\|\frac{\partial}{\partial x_{k}} u_{m}\right\|_{L^{\infty}} \leq\left\|K^{(m)}\right\|_{L^{1}}\left\|\frac{\partial}{\partial x_{k}} u_{0}\right\|_{L^{\infty}}+\sum_{\ell=1}^{m} \sum_{i, j=1}^{n}\left\|\frac{\partial^{3}}{\partial x_{i} \partial x_{j} \partial x_{k}} K^{(\ell)}\right\|_{L^{1}} \cdot \underset{\max _{i, j=1 \ldots n}\left\|f_{i j}\right\|_{L^{\infty}} .}{ }
$$

By (2.2) we obtain

$$
\left\|K^{(m)}\right\|_{L^{\infty}}=\frac{1}{(2 \pi)^{n}} \int_{\mathbb{R}^{n}}\left|\int_{\mathbb{R}^{n}} e^{-i x \cdot \xi} \varphi^{m}(\xi) d \xi\right| d x .
$$

Setting $\eta=\sqrt{\ell} \xi, y=\frac{x}{\sqrt{\ell}}$,we have that $(2.23)$ becomes

$$
\frac{1}{(2 \pi)^{n}} \int_{\mathbb{R}^{n}}\left|\int_{\mathbb{R}^{n}} e^{-i \eta \cdot y} \varphi^{m}\left(\frac{\eta}{\sqrt{\ell}}\right) d \eta\right| d y .
$$

By Lemma 4, this expression is uniformly bounded. Similarly, we can write

$$
\left\|\frac{\partial^{3}}{\partial x_{i} \partial x_{j} \partial x_{k}} K^{(m)}\right\|_{L^{1}}=\frac{1}{(2 \pi)^{n}} \int_{\mathbb{R}^{n}}\left|\int_{\mathbb{R}^{n}} e^{-i x \cdot \xi} \xi_{i} \xi_{j} \xi_{k} \varphi^{m}(\xi) d \xi\right| d x .
$$

Setting $\eta=\sqrt{\ell} \xi, y=\frac{x}{\sqrt{\ell}}$, we have that (2.25) becomes

$$
\frac{1}{(2 \pi)^{n} \ell^{3 / 2}} \int_{\mathbb{R}^{n}}\left|\int_{\mathbb{R}^{n}} e^{-i y \cdot \eta} \eta_{i} \eta_{j} \eta_{k} \varphi^{m}\left(\frac{\eta}{\sqrt{\ell}}\right) d \eta\right| d y .
$$


Therefore, according to Lemma 4, there is a positive constant such that

$$
\sum_{\ell=1}^{m}\left\|\frac{\partial^{3}}{\partial x_{i} \partial x_{j} \partial_{k}} K^{(\ell)}\right\| \leq c \sum_{\ell=1}^{m} \frac{1}{\frac{3}{2}}<+\infty .
$$

By hypothesis, the functions $f_{i j} i, j=1 \ldots n$ are bounded, hence it yields the result of the proposition.

3. Bounds for the solution of the scheme. In this section we shall prove that $u_{m}$, solutions of (1.8), have an $L^{\infty}$ bound uniformly in $m \in \mathbb{N}$.

As a first step let us state the following proposition.

Proposition 2. There is a constant $c>0$ that does not depend on $m \in \mathbb{N}$ such that

$$
\left\|u_{m+1}-u_{m}\right\|_{L^{\infty}} \leq c .
$$

where $u_{m}, m \in \mathbb{N}$ are solutions of (1.8)

Proof. In order to prove the result, notice that it holds

$$
\begin{aligned}
u_{m+1}(x)-u_{m}(x)=\int_{\mathbb{R}^{n}} K(y)\left(u_{m}(x-y)-u_{m}(x)\right) d y & \\
& +\sum_{i, j=1}^{n} \int_{\mathbb{R}^{n}} \frac{\partial^{2}}{\partial y_{i} \partial y_{j}} K(y) f_{i j}\left(u_{m}(x-y), x-y\right) d y
\end{aligned}
$$

From Proposition 1, there is a constant $c_{1}>0$ such that

$$
\left|u_{m}(x-y)-u_{m}(x)\right| \leq c_{1}|y| .
$$

Using Hölder's inequality, we can bound (3.2) as

$$
\begin{aligned}
& \left|u_{m+1}(x)-u_{m}(x)\right| \\
\leq & c_{1} \int_{\mathbb{R}^{n}}|K(y) y| d y+\max _{i, j=1 \ldots n}\left\|f_{i j}\right\|_{L^{\infty}} \sum_{i, j=1}^{n}\left\|\frac{\partial^{2}}{\partial y_{i} \partial y_{j}} K\right\|_{L^{1}} .
\end{aligned}
$$

By hypothesis $f_{i j} i, j=1 \ldots n$ are bounded and $K \in C_{c}^{3}\left(\mathbb{R}^{n}\right)$. Hence, we have obtained the result of the proposition.

Notice that for the proof of the previous result it would be enough to have $K$ with exponential decay at infinity.

Now, we can prove the main result of the paper. 
Proposition 3. There is a constant $c>0$ that does not depend on $m \in \mathbb{N}$ such that

$$
\left\|u_{m}\right\|_{L^{\infty}} \leq c
$$

where $u_{m}, m \in \mathbb{N}$ are solutions of (1.8)

Proof. Let us consider $M$ such that

$$
M>1+c_{1} a+c_{2}+\left\|u_{0}\right\|_{L^{\infty}},
$$

where $c_{1}$ is the $L^{\infty}$ bound of $\nabla u_{m}$ obtained in Proposition $1, c_{2}$ is the $L^{\infty}$ bound of $\left(u_{m+1}(x)-u_{m}(x)\right)$ obtained in Proposition 2 and $a$ is a constant such that $\operatorname{supp} K \subset B_{a}(0)$.

Now, suppose that $u_{m}, m \in \mathbb{N}$ does not have an $L^{\infty}$ bound, uniformly in $m \in \mathbb{N}$. Therefore, there exists $m_{0} \in \mathbb{N}$ and $\bar{x} \in \mathbb{R}^{n}$ such that

$$
\left|u_{m_{0}}(\bar{x})\right| \geq M \quad \text { and } \sup _{m \leq m_{0}-1}\left\|u_{m}\right\|_{L^{\infty}}<M .
$$

From the definition of $u_{m_{0}}$ we have

$$
\left|u_{m_{0}}(\bar{x})\right| \leq\left|K_{*} u_{m_{0}-1}(\bar{x})\right|+\left|\left(K_{*} \sum_{i, j=1}^{n} \frac{\partial^{2}}{\partial x_{i} \partial x_{j}} f_{i j}\left(u_{m_{0}-1}, \cdot\right)\right)_{(\bar{x})}\right| .
$$

From (1.2), (3.7) and using Hölder's inequality, it follows that

$$
\left|K_{*} u_{m_{0}-1}(\bar{x})\right| \leq\|K\|_{L^{1}}\left\|u_{m_{0}-1}\right\|_{L^{\infty}}<M .
$$

From Proposition 1 we obtain

$$
\left|u_{m_{0}-1}(\bar{x})-u_{m_{0}-1}(x)\right| \leq c_{1}|x-\bar{x}|, \quad \forall x \in \mathbb{R}^{n} .
$$

From Proposition 2 we have

$$
\left|u_{m_{0}}(\bar{x})-u_{m_{0}-1}(\bar{x})\right| \leq c_{2}, \quad \forall x \in \mathbb{R}^{n} .
$$

Hence, we get

$$
\left|u_{m_{0}-1}(x)\right| \geq\left|u_{m_{0}-1}(\bar{x})\right|-c_{2}-c_{1}|x-\bar{x}| \geq\left|u_{m_{0}}(\bar{x})\right||x-\bar{x}|, \quad \forall x \in \mathbb{R}^{n} .
$$

From (3.6) and (3.7) we have

$$
\left|u_{m_{0}-1}(\bar{x})\right|-c_{2}-c_{1}|x-\bar{x}| \geq\left|u_{m_{0}}(\bar{x})\right||x-\bar{x}|>1, \quad \forall x \in B_{a}(\bar{x}) .
$$

This combined with the assumption (1.4) for $f_{i j}$ yields that

$$
\frac{\partial^{2}}{\partial x_{i} \partial x_{j}} f_{i j}\left(u_{m_{0}-1}(x), x\right) \equiv 0, \quad \forall x \in B_{a}(\bar{x}), i, j=1 \ldots n .
$$

Now, since $\operatorname{supp} K \subset B_{a}(0)$ we have that

$$
\left(K_{*} \sum_{i, j=1}^{n} \frac{\partial^{2}}{\partial x_{i} \partial x_{j}} f_{i j}\left(u_{m_{0}-1}, \cdot\right)\right)(\bar{x})=0
$$

In view of (3.9) and (3.15), we have obtained $\left|u_{m_{0}}(\bar{x})\right|<M$, which is a contradiction with (3.7). 
Acknowledgements. I would like to thank Luis A. Caffarelli for his suggestions and comments.

\section{REFERENCES}

[CM] L. A. Caffarelli And N. E. Muler, An $L^{\infty}$ bound for solutions of the CahnHilliard Equation, Arch. rational Mech. Anal. 133 (1995), 129-144.

[C] J. W. CAHn, On spinodal decomposition, Acta Metall. 9 (1961), 795-801.

[D] R. Durrett, Probability, Theory and Examples, Wadsworth and Brooks, 1991.

[Fe] W. Feller, An Introduction to Probability Theory and Its Applications, John Wiley, 1968.

[HN] K. Hollig AND J. NoHEL, A diffusion equation with a nonmonotone constitutive function, System of Nonlinear Partial Differential Equations (1983), 409-422.

[P] R. L. PEgO, Front migration in the nonlinear Cahn-Hilliard equation, Proc. R. Soc. London 422 (1989), 261-278.

[S] M. Slemrod, Dynamics of measured value solutions to a backward-forward heat equation, J. Dynamics and Differential Equations 3 (1991), 1-28.

Departamento de Matemática

Facultad de Ciencias Exactas

Universidad Nacional de Buenos Aires

Ciudad Universitaria

(1428) Buenos Aires, República Argentina

Received: December 20th, 1995. 